\title{
Algunas ideas de investigación científica
}

\author{
Calizaya José Manuel ${ }^{1}$ \\ ORCID: https://orcid.org/0000-0001-6221-0909 \\ jcalizayal@unsa.edu.pe \\ Universidad Nacional de San Agustín de Arequipa
}

Arequipa-Perú

Recibido (01/07/20), Aceptado (17/07/20)

Resumen: La investigación científica parte de un precepto fundamental que es la existencia de una estructura lógica, y por ende no contradictoria, que articula de manera fluida a todo el conocimiento humano que ha sido verificado, racionalizado y conceptualizado en un área determinada; a esto se le denomina ciencia. En este trabajo se presenta un análisis teórico de la investigación científica desde un punto de vista social.

Palabras Clave: Investigación científica, investigación social, conocimiento humano.

\section{Some Scientific Research Ideas}

\begin{abstract}
Scientific research starts from a fundamental precept that is the existence of a logical structure, and therefore not contradictory, that fluidly articulates all human knowledge that has been verified, rationalized and conceptualized in a given area; this is called science. This work presents a theoretical analysis of scientific research from a social point of view.
\end{abstract}

Keywords: scientific research, social research, human knowledge. 


\section{I.INTRODUCCIÓN}

Ninguna investigación científica puede hacerse sin las personas que dedican su tiempo y esfuerzo a la confirmación o refutación de teorías científicas, a la búsqueda de nuevos conocimientos o a la ampliación de los ya existentes; a estas personas se les suele conocer como investigadores o científicos. Los investigadores pueden apoyarse, según sus propósitos en alguno de los dos, o en ambos (enfoque mixto) enfoques clásicos a conocer: el enfoque cualitativo y el enfoque cuantitativo.

En el enfoque cualitativo, conocido también enfoque fenomenológico trata de estudiar un problema sin extraerlo de su entorno, analizando sus interacciones para buscar patrones que modelen su comportamiento [1]. En las ciencias sociales la metodología cualitativa toma como modelos la teoría crítica que estudia las estructuras sociales imbricadas como la política, los grupos étnicos y la economía entre otros, y el constructivismo que busca reconstruir el camino que modeló al problema estudiado.

El enfoque cuantitativo por otro lado parte del paradigma explicativo del positivismo, donde el acercamiento a la investigación de un problema se realiza desde un punto de vista racional, objetivo, que permite la observación, manipulación y verificación de predicciones acerca del mismo [2]. La verificación de postulados e hipótesis muestran la validez o falsedad de los mismos, por lo tanto, cualquier problema planteado debe ser susceptible a comprobaciones experimentales que prueben su validez y de esta manera pueda ser aceptado como parte constituyente de una teoría formal [3]. El enfoque cualitativo tiene su origen las investigaciones científicas de las ciencias naturales, que luego fueron adoptadas por las ciencias sociales con bastante éxito y han demostrado además ser de mucha utilidad en este ramo. Esta es la metodología que se usará en este trabajo y a partir de ella se desarrollarán las directrices que son necesarias para la ejecución de cualquier proyecto de investigación científica en ciencias sociales.

\section{II.DESARROLLO}

La finalidad de la investigación científica consiste en verificar, rebatir, ampliar o proponer nuevos aportes experimentales o teóricos al conjunto de la ciencia que se están abordando. La formulación de un problema de investigación científica radica en la generación propia de los investigadores, sobre sus experiencias y experticias en el área de estudio. Estos estarán sujetos a los aportes que el grupo de investigadores decida realizar al campo del conocimiento [4]. Esta premisa implica el investigador está inmerso en un proceso continuo de actualización en lo que respecta a los aportes que se están haciendo a esa línea de conocimiento particular. El investigador a menudo desarrolla su trabajo partiendo de su formación teórica y metodológica, por lo que sus inquietudes investigativas lo direccionan hacia la ampliación del conocimiento partiendo de una línea de investigación, la cual también le une a una comunidad de investigadores con inclinaciones y temas investigativos similares.

La investigación científica no se establece solo entre investigadores experimentados, sino que da cabida a nuevos entusiastas de la ciencia y el conocimiento. Para aquellos investigadores que se inician en el proceso de creación o ampliación de conocimiento científico se recomienda un grado de inmersión bibliográfica más profunda como también la guía y apoyo de investigadores que, con una formación consolidada pueden servir de mentores y apoyar su proceso de crecimiento en el área [5]. En última instancia se recomienda una aproximación gradual a los temas de investigación que deseen desarrollar a fin de que las competencias requeridas para realizar trabajos de calidad científica estén acorde al nivel del conocimiento que domina el nuevo investigador [6].

La figura 1 muestra los aspectos más resaltantes de un proceso de investigación, en ella se evidencian elementos esenciales para ejecutar la investigación de forma asertiva. Se observa que el problema puede tener diferentes enfoques según las necesidades y gustos del investigador, sin embargo en cualquiera de los casos éste debe tener un alcance [7], que será la parte fundamental para definir el problema y focalizar la investigación en ese respecto. 


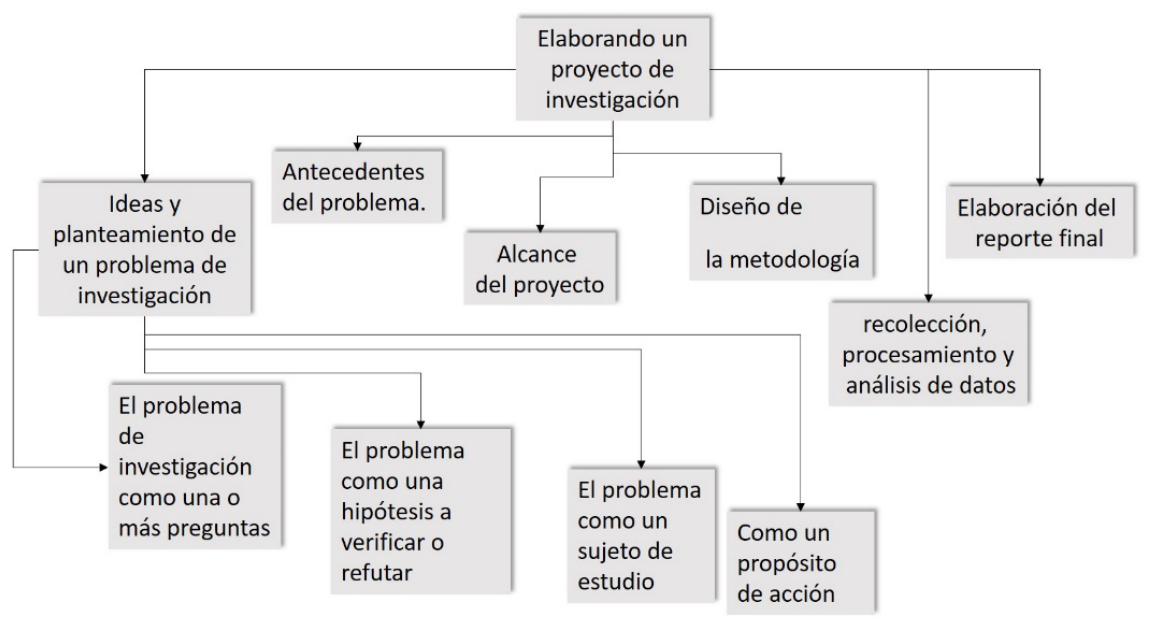

Figura 1. Elementos básicos de una investigación.

\section{A.Análisis de los posibles problemas de investigación.}

El acercamiento a un problema de investigación requiere en un principio de una evaluación metodológica, de pensar cómo abordar el problema en sí para definir qué estrategias y recursos deben ser utilizados a fin de poder estudiarlo de manera satisfactoria. En este libro se presentará cómo aproximarse a un problema de interés social utilizando la metodología de investigación cualitativa. El desarrollo de cualquier problemática en este campo se realizará mediante esta metodología.

La investigación social cuantitativa nace de las investigaciones y aportes teóricos de Auguste Comte y Emile Durkheim [8], los padres del positivismo, y quienes concluyeron que los fenómenos sociales pueden ser estudiados utilizando el mismo método que utilizan las ciencias naturales, que es el ya mencionado y descrito método científico.

Sin embargo, en la actualidad el enfoque cuantitativo para las ciencias sociales está más adscrito a las corrientes pospositivistas que permiten una mayor flexibilidad al acercarse a un problema concreto. En este último enfoque se pasa del conocimiento como copia de la realidad objetiva, a la del conocimiento como producto de la interacción que existe entre el conocedor y el sujeto o problema conocido.

\section{B.Formulando el proyecto de investigación.}

Un proyecto de investigación es un trabajo documentado donde se plasma el proceso que inicia con el acercamiento al problema que se desea investigar hasta los resultados obtenidos de las experiencias elaboradas para tal fin. El proceso comprende una serie de etapas que registran de qué manera se trató el problema y cuáles fueron sus resultados y consecuencias. Los componentes o etapas de un proyecto de investigación social cuantitativo se pueden mostrar de la siguiente manera:

a)Ideas y planteamiento de un problema de investigación

b)Revisión del soporte teórico, bases y fundamentos: Antecedentes del problema.

c)Alcance del proyecto: Objetivos de la investigación

d)Diseño de la metodología

e)Diagrama de trabajo: recolección, procesamiento y análisis de datos.

f)Elaboración del reporte final.

Todo proyecto de investigación social inicia con una idea de investigación, que tiene como propósito el encontrar una respuesta o solución a un problema que permita el avance, la ampliación, la refutación o verificación de un conocimiento en las ciencias sociales. La idea de investigación indica qué es aquello que se busca investigar y frecuentemente engloba un conjunto de temas que luego van siendo acotados hasta llegar a la identificación del problema a ser abordado para la búsqueda de su solución.

Los problemas que abordan la investigación en ciencias sociales pueden ir desde una situación social determi- 
nada como las ventajas y desventajas de la inmigración venezolana en Colombia, la optimalización del aprendizaje bajo condiciones de pandemia en Perú, o pueden ser acercamientos teóricos como, por ejemplo, cuáles factores eternizan el populismo en américa latina, la precariedad de los sindicatos, la debilidad de las democracias modernas, la brecha digital en américa, entre otros.

Los antecedentes en la investigación social, tienen la importancia de ubicar la investigación dentro del campo al cual pertenece, destacando los aportes que otros investigadores han alcanzado sobre el tema que se busca desarrollar, en el caso particular de una investigación de tipo social, pueden incluirse antecedentes referentes al problema de estudio como antecedentes referentes al proceso ejecutado para realizar el estudio, pues al tratarse de temas sociales, son muchos los factores que pudiesen influir en la conducta humana y su repercusión a lo que se busca en la investigación.

Con los objetivos definidos, el investigador debe elaborar la metodología necesaria para resolver el problema o los problemas planteados. Dentro de la metodología destacan las estrategias y las técnicas que le permitirán alcanzar dichos objetivos, comprobando y verificando si coinciden con los supuestos teóricos esperados.

El diseño metodológico contempla la manera adecuada de recolectar las muestras o datos necesarios para llevar a cabo la investigación, especificando los procedimientos con detalle a fin de no introducir anomalías en los mismos.

Una vez concebida la aproximación metodológica que dará respuestas al problema planteado según unos objetivos definidos, toca el momento de desarrollar las etapas de recolección, procesamiento y análisis de la información, la misma que estará constituida por las muestras o datos que se utilizaran para tal fin.

La recolección de datos comprende tres partes fundamentales que le dan fuerza y solidez a la investigación. Estas se describen en la figura 2, y debe destacarse que son las partes más importantes en el proceso de investigación, pues de ellas depende el nivel profesional con el que se ha desarrollado la investigación, además permite conocer qué se logró con el proceso de la investigación y cuáles podrían ser las directrices para futuras investigaciones.

\begin{tabular}{|c|c|c|c|}
\hline $\begin{array}{c}\text { Recolección } \\
\text { de datos }\end{array}$ & $\rightarrow \begin{array}{c}\text { Procesamiento } \\
\text { de datos }\end{array}$ & $\rightarrow$ & $\begin{array}{c}\text { Análisis de } \\
\text { datos }\end{array}$ \\
\cline { 2 - 3 }
\end{tabular}

Figura 2. Fases de la recolección de datos.

\section{C.Presentación final del documento}

Una vez cumplidos todos los procesos para dar por culminada la investigación, es importante que se elabore un informe o un artículo científico que contribuya a la divulgación de los resultados encontrados y aporte al área de estudio. En este punto ya se ha procedido al análisis e interpretación de los resultados que arrojó la investigación. Ya se cotejaron los resultados experimentales con los objetivos buscados, verificándose si los postulados o las hipótesis se cumplieron o no y qué consecuencias generan dichos resultados, cómo se relacionan con el trabajo de otros investigadores y que aportan al acerbo científico del área investigada.

\section{III.CONCLUSIONES}

El trabajo de investigación es un proceso que debe seguir normas claras y éticas para que se ejecute de la forma correcta y pueda brindar resultados satisfactorios que aporten a la comunidad científica de estudio.

Cumplir con una buena definición del problema puede ser suficiente para poder encaminar un proyecto de investigación exitoso, sin embargo es necesario incorporar elementos que fortalezcan este planteamiento y puedan conducir a los resultados esperados.

La formulación crítica y objetiva de los resultados es indispensable para poder realizar el informe final, que brinde un documento científico apropiado para ofrecer un aporte al área de estudio, tal que pueda generar nuevas investigaciones y nuevos complementos para la investigación.

\section{REFERENCIAS}

[1]R. Hernández-Sampieri, Metodología de la investigación, Mexico: Mc Graw Hill, 2018.

[2]L. Blaxter, C. Hughes y M. Tight, Cómo se hace una investigación., Barcelona: Gedisa, 2000. 
[3]F. Alvira, «La perspectiva cualitativa y cuantitativa en las investigaciones sociales,» Revista estudios de psicología,

no 11, pp. 34-39, 1982.

[4]C. Monje Álvarez, Metodología de la Investigación Cuantitativa y Cualitativa,, Colombia: Universidad SurColombiana:

Facultad de Ciencias Sociales y Humanas, 2011.

[5]Briones, Métodos y Técnicas de Investigación para las Ciencias Sociales., Colombia: Colombia: Intituto Colombiano para el Fomento de la educación suprior ICFES, 1996.

[6]M. C. Müggenburg Rodríguez y I. Pérez Cabrera, «Tipos de estudio en el enfoque de investigación cuantitativa,»

Enfermería Universitaria, vol. 4, $\mathrm{n}^{\mathrm{o}}$ 1, p. 35.42, 2007.

[7]M. Amakforoosh, Metodología de la investigación., México, D.F.: Editorial Limusa, 2001.

[8]N. López y I. Sandoval, «Métodos y técnicas de investigación cuantitativa y cualitativa.,» [En línea]. Available: http://recursos.udgvirtual.udg.mx/biblioteca/bitstream/20050101/1103/1/

Metodos_y_tecnicas_de_investigacion_cuantitativa_y_cualitativa.pdf. [Último acceso: 09 noviembre 2020].

\section{RESUMEN CURRICULAR}

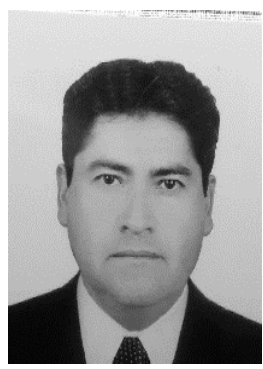

Calizaya López, José Manuel, Doctor en Ciencias de la Educación, Magíster en Ciencias con Mención en Gestión Social - Desarrollo Sostenible y Licenciado en Trabajo Social. Docente Principal y Director del Departamento Académico de Trabajo Social, Docente investigador UNSA- INVESTIGA y miembro del Instituto de Investigaciones Sociales de la Universidad Nacional de San Agustín, Arequipa, Perú. 